\title{
Vector hysteresis modeling coupled with a loop-based magnetic equivalent circuit
}

\section{Citation for published version (APA):}

Ceylan, D., Zeinali, R., Daniels, B., Boynov, K. O., \& Lomonova, E. A. (2021). Vector hysteresis modeling coupled with a loop-based magnetic equivalent circuit. In 2021 16th International Conference on Ecological Vehicles and Renewable Energies, EVER 2021 [9456642] Institute of Electrical and Electronics Engineers. https://doi.org/10.1109/EVER52347.2021.9456642

DOI:

10.1109/EVER52347.2021.9456642

Document status and date:

Published: 23/06/2021

\section{Document Version:}

Accepted manuscript including changes made at the peer-review stage

\section{Please check the document version of this publication:}

- A submitted manuscript is the version of the article upon submission and before peer-review. There can be important differences between the submitted version and the official published version of record. People interested in the research are advised to contact the author for the final version of the publication, or visit the $\mathrm{DOI}$ to the publisher's website.

- The final author version and the galley proof are versions of the publication after peer review.

- The final published version features the final layout of the paper including the volume, issue and page numbers.

Link to publication

\section{General rights}

Copyright and moral rights for the publications made accessible in the public portal are retained by the authors and/or other copyright owners and it is a condition of accessing publications that users recognise and abide by the legal requirements associated with these rights.

- Users may download and print one copy of any publication from the public portal for the purpose of private study or research.

- You may not further distribute the material or use it for any profit-making activity or commercial gain

- You may freely distribute the URL identifying the publication in the public portal.

If the publication is distributed under the terms of Article 25fa of the Dutch Copyright Act, indicated by the "Taverne" license above, please follow below link for the End User Agreement:

www.tue.nl/taverne

Take down policy

If you believe that this document breaches copyright please contact us at:

openaccess@tue.nl

providing details and we will investigate your claim. 


\title{
Vector Hysteresis Modeling Coupled with a Loop-based Magnetic Equivalent Circuit
}

\author{
Doğa Ceylan, Reza Zeinali, Bram Daniels, Konstantin O. Boynov, and Elena A. Lomonova \\ Department of Electrical Engineering, Eindhoven University of Technology, 5612 AZ Eindhoven, The Netherlands \\ Email: d.ceylan@tue.nl
}

\begin{abstract}
This paper proposes a method to include the anisotropic magnetic hysteresis property of the softmagnetic material in the magnetic equivalent circuit (MEC) modeling. The loop-based MEC formulation is improved to handle the non-linearity of the anisotropic magnetic hysteresis. Single MEC element of NO27 electrical steel is modeled in 2-D under both rotating and alternating magnetic fields to achieve an accurate iron loss estimation for different excitation frequencies. The developed model is coupled with both the single-valued $B H$ curve (SVC) and the hysteresis $B H$ loop (HL) of the non-linear magnetic material. The magnetic flux density is calculated as the output of the model and used for the comparison of both magnetic saturation calculations. The results show that an alternating magnetic field in a single direction with a peak value smaller than $300 \mathrm{~A} / \mathrm{m}$ causes a discrepancy of more than $10 \%$ between HL and SVC, where the knee point of the SVC is located at $200 \mathrm{~A} / \mathrm{m}$. Although the SVC approximation gives realistic results under the deep magnetic saturation, it is not capable of estimating the iron loss accurately. The proposed model with the HL calculates the core loss density as 4 and $28 \mathrm{~W} / \mathrm{kg}$ for $1000 \mathrm{~A} / \mathrm{m}$ peak value with 50 and $200 \mathrm{~Hz}$ alternating magnetic field excitation, respectively.
\end{abstract}

Index Terms-Magnetic vector hysteresis, magnetic equivalent circuit, fixed-point method, iron loss estimation.

\section{INTRODUCTION}

The design of efficient electromagnetic devices with a soft-magnetic material requires an accurate iron loss estimation. The most commonly used method to model the local magnetic saturation is coupling the finite element method (FEM) with a non-linear solver based on the single-valued $B H$ curve (SVC) of the softmagnetic material as in [1]. Although using the SVC approximation to define the magnetic saturation is computationally effective, it suffers from accuracy depending on the magnitude and frequency of the excitation. In particular, a magnetic field excitation that results in minor loops inside the major hysteresis loop of the soft-magnetic material decreases the accuracy of the
SVC approximation. A static hysteresis modeling is implemented with the finite element formulation in [2]. However, a large number of mesh elements in FEM cause to increase the computational cost and even to lose the convergence of the nonlinear solver, when the FEM model is coupled with the magnetic hysteresis $\mathrm{BH}$ loop (HL). The magnetic equivalent circuit (MEC) modeling is an alternative to FEM for the analysis of electromagnetic devices with a soft-magnetic material. MEC is a differential scheme that approximates the Taylor series expansion in terms of the difference between two point values, while FEM is an integral method that constructs the solution in each element from the basis functions. FEM is capable of handling extremely complicated, time-dependent geometries. However, the differential MEC can find the global solution with less number of elements than the integral FEM needs, if the geometry of the electromagnetic problem is represented with regular brick-shaped elements. [3] shows that MEC is more stable in the calculation of the 2-D motional eddy currents with regular elements in comparison with FEM.

In [4], an equivalent magnetic circuit is integrated into the electrical equivalent circuit of a magnetic device used in the drive electronics in terms of lumped parameters. The proposed method in [4] models the magnetic resistance using the measured major hysteresis loop of the soft-magnetic material without considering the minor loops or the anisotropy of the magnetic hysteresis. Moreover, a generalized simple equivalent electrical circuit is proposed to solve the Cauchy problem for the nonlinear hysteric phenomenon in [5]. Equivalent circuit modeling of a hysteresis interior permanent magnet motor is investigated in [6], where the Elliptical modeling approximation is used to model the major hysteresis loop. [7] recently proposes a Cauer's equivalent circuit to present dynamic hysteresis characteristics instead of solving it with MEC. Finally, a significant contribution to model the magnetic hysteresis with MEC is presented in [8], where two non-linear solvers are employed in series: 
the Newton-Raphson and fixed-point methods. In the first part of the proposed algorithm, the Newton-Raphson method is used to calculate the magnetic flux density based on the SVC. In the second part, the calculated flux density distribution is updated with the fixed-point method using a Preisach hysteresis model.

In this study, the formulation of the loop-based MEC model used in [9] is improved to couple the existing MEC model with the anisotropic vector hysteresis model presented in [10]. The system of equations of the coupled model is solved iteratively using only the fixed-point method for a single MEC element under a magnetic field with various magnitudes and frequencies. Both models and the coupling are discussed in Section II, while the results with HL and SVC are compared in Section III.

\section{Modeling Method}

The local magnetic saturation is modeled in 2-D using the loop-based magnetic equivalent circuit (MEC) coupled with a vector hysteresis model. The non-linear hysteric problem is solved iteratively using the fixedpoint method as implemented in [11] for an SVC.

\section{A. Loop-based Magnetic Equivalent Circuit Model}

There exist two types of MEC modeling techniques in the literature: node-based (also known as nodal-based), and loop-based (also known as mesh-based) MEC. A comparative study between both MEC techniques is presented in [12], where the loop-based model is found to be significantly better than the node-based model under nonlinear operation. The loop-based MEC uses Kirchhoff's voltage law to calculate its pseudo flux loops. The authors have recently used this method to model the iron parts of a variable flux reluctance machine [9]. The magnetic saturation is modeled in MEC using two different approaches: the single-valued $B H$ curve (SVC) and the vector hysteresis $B H$ loop (HL).

\section{B. MEC with the Single-valued BH Curve}

The first-order single-variable Taylor series expansion is used to model SVC:

$$
H=f(B)=f\left(B_{0}\right)+f^{\prime}\left(B_{0}\right)\left(B-B_{0}\right),
$$

where $f$ represents the SVC, which takes the magnetic flux density $(B)$ as input and gives the magnetic field strength $(H)$ as output. (1) is re-written in a tangent line format:

$$
H=f^{\prime}\left(B_{0}\right) B+f\left(B_{0}\right)-f^{\prime}\left(B_{0}\right) B_{0} .
$$

The slope and intercept of the tangent line of $f$ are called the reluctivity, which is one over permeability $\left(v=\mu^{-1}\right)$, and the coercivity $\left(H_{c}\right)$, respectively:

$$
\mu=f^{\prime}\left(B_{0}\right)^{-1} \text {, and } H_{c}=f\left(B_{0}\right)-f^{\prime}\left(B_{0}\right) B_{0} .
$$

Hence, $\mu$ and $H_{c}$ are calculated iteratively using the output of MEC $\left(B_{0}\right)$, and the SVC $(f)$ as in (3) based on the fixed-point algorithm. Although the MEC with an SVC uses only one permeability $(\mu)$ and one coercivity $\left(H_{c}\right)$ for each element depending on the modulus of the magnetic flux density of that element, the magnetic saturation in $x$ - and $y$-directions is approximated as

$$
\left[\begin{array}{l}
H_{x} \\
H_{y}
\end{array}\right]=\left[\begin{array}{cc}
\mu^{-1} & 0 \\
0 & \mu^{-1}
\end{array}\right]\left[\begin{array}{l}
B_{x} \\
B_{y}
\end{array}\right]+\frac{1}{|B|}\left[\begin{array}{l}
H_{c} B_{x} \\
H_{c} B_{y}
\end{array}\right] .
$$

Fig. 1(a) shows the structure of a single MEC element with the SVC. $\mathcal{R}, \mathcal{F}^{e x}$, and $\mathcal{F}^{B H}$ refer to the reluctance, the excitation related magneto-motive force (MMF), and the magnetic saturation related MMF, respectively. Lumped parameters in the positive side of the $x$-axis of the MEC element with SVC are expressed using

$$
\begin{gathered}
\mathcal{R}_{x+}=\frac{l_{x+}}{l_{y} l_{s} \mu}, \\
\mathcal{F}_{x+}^{e x}=\left|H^{e x}\right| \cos \left(\angle H^{e x}\right) l_{x+}, \\
\mathcal{F}_{x+}^{B H}=-\frac{B_{x}}{|B|} H_{c} l_{x+},
\end{gathered}
$$

where $l_{x+}$ is the length of the positive part of the MEC element in $x$-direction, $l_{y}$ is the total length in $y$-direction, $l_{s}$ is the stack length of the MEC element, $H^{e x}$ is the magnetic field excitation applied to the MEC element. Equations for other lumped parameters of the MEC element with SVC are derived with the same approach.

\section{MEC with the Vector Hysteresis BH Loop}

The anisotropic congruency-based vector hysteresis model presented in [10] is employed in this study to be coupled with the loop-based MEC described in Section A. The anisotropy of the hysteresis model is achieved by combining two scalar hysteresis models which are developed by first-order reversal curves of NO27 soft-magnetic material measured parallel and perpendicular to the rolling direction. Then, the developed anisotropic scalar model is extended to a vector hysteresis model based on Mayergoyz vector hysteresis generalization. Details of the used vector hysteresis model and its verification with measurements are reported by the authors in [10]. Unlike (1) of SVC, the magnetic saturation in the model with HL is expressed separately in both directions:

$$
H_{x}=f_{1}\left(B_{x}, B_{y}\right) \text {, and } H_{y}=f_{2}\left(B_{x}, B_{y}\right) .
$$

Therefore, it requires to use two separate first-order twovariable Taylor series expansions for $x$ - and $y$-directions:

$$
\begin{aligned}
H_{x}= & f_{1}\left(B_{x_{0}}, B_{y_{0}}\right)+\frac{\partial f_{1}\left(B_{x_{0}}, B_{y_{0}}\right)}{\partial x}\left(B_{x}-B_{x_{0}}\right) \\
& +\frac{\partial f_{1}\left(B_{x_{0}}, B_{y_{0}}\right)}{\partial y}\left(B_{y}-B_{y_{0}}\right),
\end{aligned}
$$




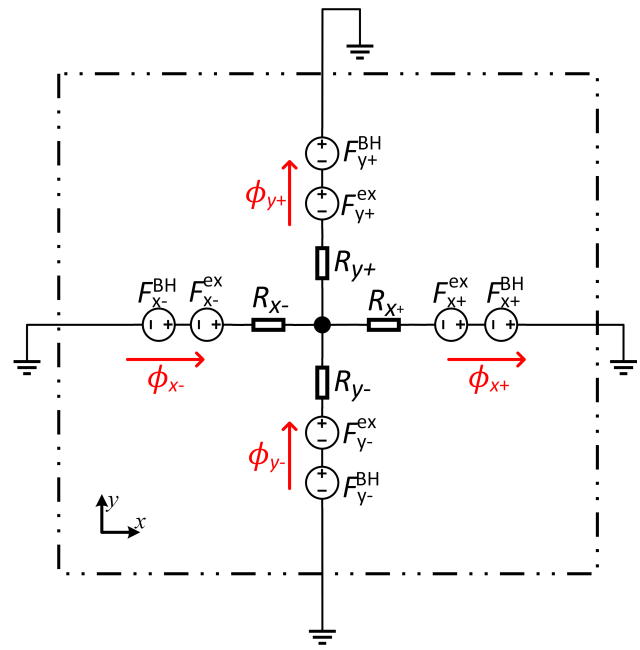

(a) A MEC element with SVC

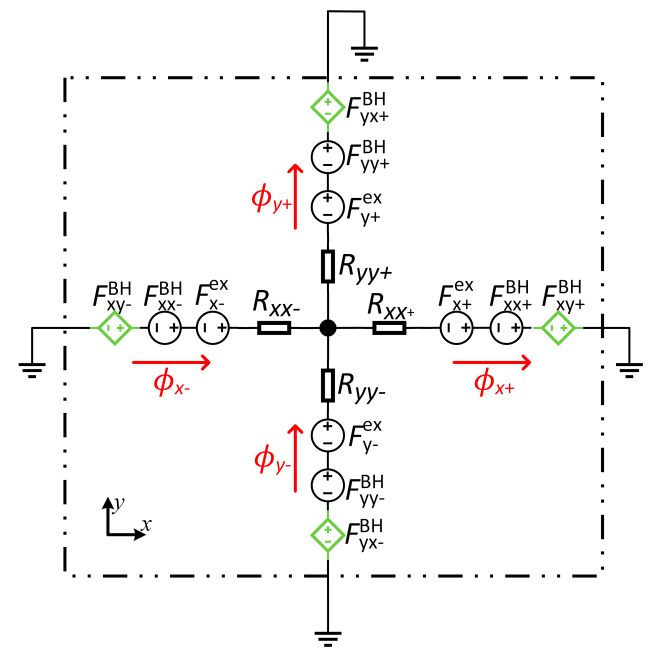

(b) A MEC element with vector HL

Fig. 1: Single MEC element with its lumped circuit parameters to be used with a single-valued $B H$ curve (SVC) and a vector hysteresis $B H$ loop.

$$
\begin{aligned}
H_{y}= & f_{2}\left(B_{x_{0}}, B_{y_{0}}\right)+\frac{\partial f_{2}\left(B_{x_{0}}, B_{y_{0}}\right)}{\partial x}\left(B_{x}-B_{x_{0}}\right) \\
& +\frac{\partial f_{2}\left(B_{x_{0}}, B_{y_{0}}\right)}{\partial y}\left(B_{y}-B_{y_{0}}\right) .
\end{aligned}
$$

Then, the Taylor expansions are modified to represent the tangent lines as implemented for the SVC:

$$
\begin{aligned}
H_{x}= & \frac{\partial f_{1}}{\partial x} B_{x}+\frac{\partial f_{1}}{\partial y} B_{y} \\
& -\frac{\partial f_{1}}{\partial x} B_{x_{0}}-\frac{\partial f_{1}}{\partial y} B_{y_{0}}+f_{1}\left(B_{x_{0}}, B_{y_{0}}\right), \\
H_{y}= & \frac{\partial f_{2}}{\partial x} B_{x}+\frac{\partial f_{2}}{\partial y} B_{y} \\
& -\frac{\partial f_{2}}{\partial x} B_{x_{0}}-\frac{\partial f_{2}}{\partial y} B_{y_{0}}+f_{2}\left(B_{x_{0}}, B_{y_{0}}\right) .
\end{aligned}
$$

The slopes of the tangent lines in (11) and (12) include two self-permeabilities $\left(\mu_{x x}\right.$ and $\left.\mu_{y y}\right)$, two mutual permeabilities $\left(\mu_{x y}\right.$ and $\left.\mu_{y x}\right)$, while the intercepts define the coercivities in both directions $\left(H_{c_{x}}\right.$ and $\left.H_{c_{y}}\right)$. Therefore, (11) and (12) are combined to get

$$
\left[\begin{array}{l}
H_{x} \\
H_{y}
\end{array}\right]=\left[\begin{array}{ll}
\mu_{x x}^{-1} & \mu_{x y}^{-1} \\
\mu_{y x}^{-1} & \mu_{y y}^{-1}
\end{array}\right]\left[\begin{array}{l}
B_{x} \\
B_{y}
\end{array}\right]+\left[\begin{array}{c}
H_{c_{x}} \\
H_{c_{y}}
\end{array}\right] .
$$

To find the expressions for the lumped circuit parameters of the MEC element given in Fig. 1(b), both sides of (13) are multiplied by $\left[l_{x} l_{y}\right]^{-1}$ :

$$
\begin{aligned}
{\left[\begin{array}{c}
\mathcal{F}_{x}^{e x} \\
\mathcal{F}_{y}^{e x}
\end{array}\right]=} & {\left[\begin{array}{ll}
l_{x}\left(\mu_{x x} l_{y} l_{s}\right)^{-1} & l_{x}\left(\mu_{x y} l_{x} l_{s}\right)^{-1} \\
l_{y}\left(\mu_{y x} l_{y} l_{s}\right)^{-1} & l_{y}\left(\mu_{y y} l_{x} l_{s}\right)^{-1}
\end{array}\right]\left[\begin{array}{l}
\phi_{x} \\
\phi_{y}
\end{array}\right] } \\
& +\left[\begin{array}{l}
l_{x} H_{c_{x}} \\
l_{y} H_{c_{y}}
\end{array}\right] .
\end{aligned}
$$

To be used in Kirchhoff's law, (14) is re-written in terms of the reluctance, the flux, and the MMF:

$$
\left[\begin{array}{l}
\mathcal{F}_{x}^{e x} \\
\mathcal{F}_{y}^{e x}
\end{array}\right]=\left[\begin{array}{ll}
\mathcal{R}_{x x} & \mathcal{R}_{x y} \\
\mathcal{R}_{y x} & \mathcal{R}_{y y}
\end{array}\right]\left[\begin{array}{l}
\phi_{x} \\
\phi_{y}
\end{array}\right]+\left[\begin{array}{l}
\mathcal{F}_{x x}^{B H} \\
\mathcal{F}_{y y}^{B H}
\end{array}\right]
$$

The main difference between two MEC elements modeled with SVC and HL in Fig. 1 is the green colored flux-dependent MMF sources which represent the mutual saturation. Lumped circuit parameters in the positive side of the $x$-axis of the MEC element with HL are expressed using

$$
\begin{gathered}
\mathcal{R}_{x x+}=\frac{l_{x+}}{l_{y} l_{s} \mu_{x x}}, \\
\mathcal{F}_{x x+}^{B H}=-H_{c_{x}} l_{x+}, \\
\mathcal{F}_{x y+}^{B H}=-\mathcal{R}_{x y+} \phi_{y},
\end{gathered}
$$

where the mutual reluctance $\left(\mathcal{R}_{x y+}\right)$ and the magnetic flux in $y$-direction $\left(\phi_{y}\right)$ are calculated using

$$
\mathcal{R}_{x y+}=\frac{l_{x+}}{l_{x} l_{s} \mu_{x y}}, \text { and } \phi_{y}=\frac{\phi_{y+}+\phi_{y-}}{2} .
$$

Other lumped circuit parameters of the MEC element coupled with HL are derived using the same approach.

The fixed-point method is employed for both SVC and HL. In the developed fixed-point algorithm, the loopbased MEC calculates the magnetic flux density which is used by the non-linear magnetic characteristics of the material (SVC or HL) to calculate the permeability and the coercivity. In the next fixed-point iteration, the calculated permeability and coercivity values are used to update the related lumped circuit parameters of the MEC element.The convergence is decided by checking the relative error in the modulus of the magnetic flux density between the successive iterations. 


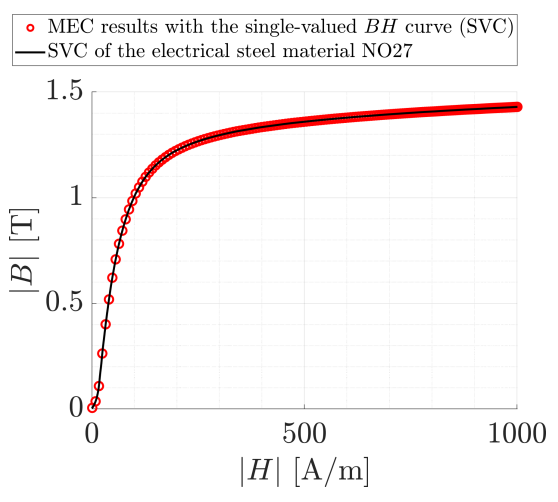

(a)

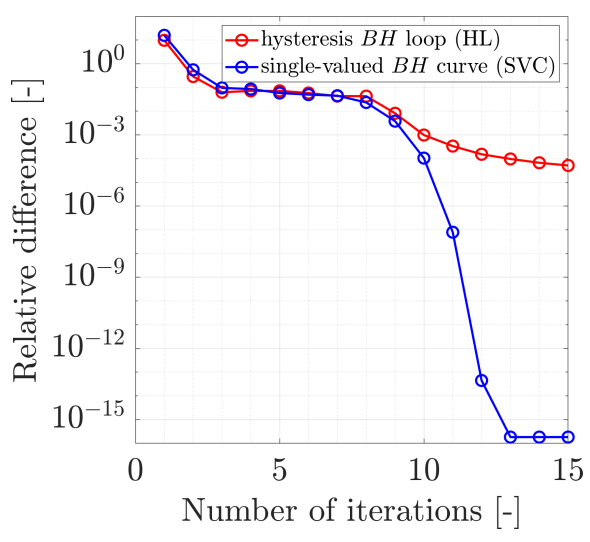

(b)

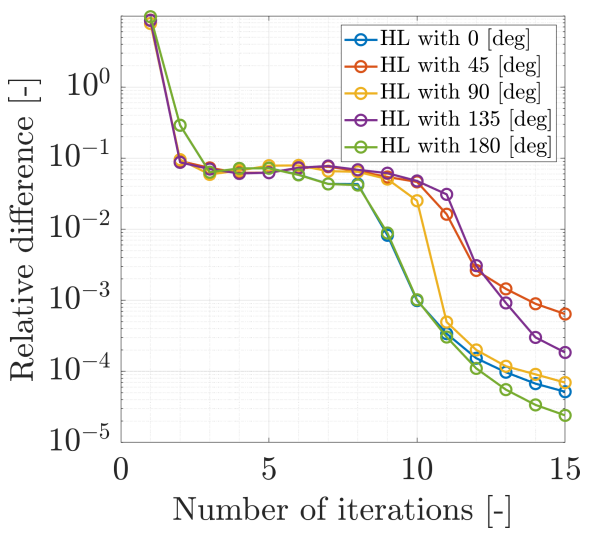

(c)

Fig. 2: (a) Verification of the model with SVC, (b) convergence comparison between models with SVC and HL, (c) convergence of the model with HL for different excitation angles.

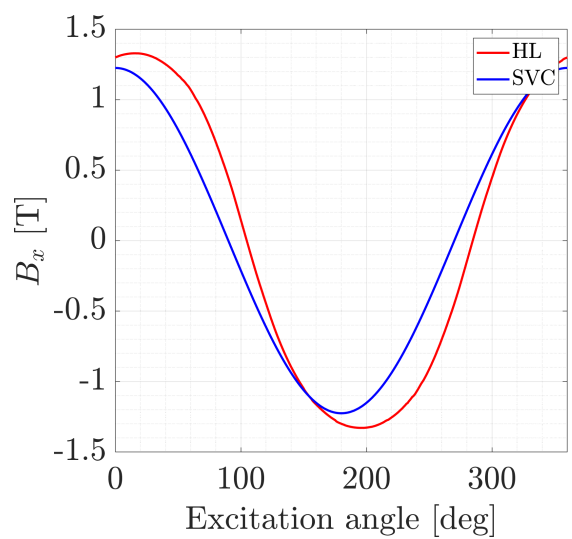

(a)

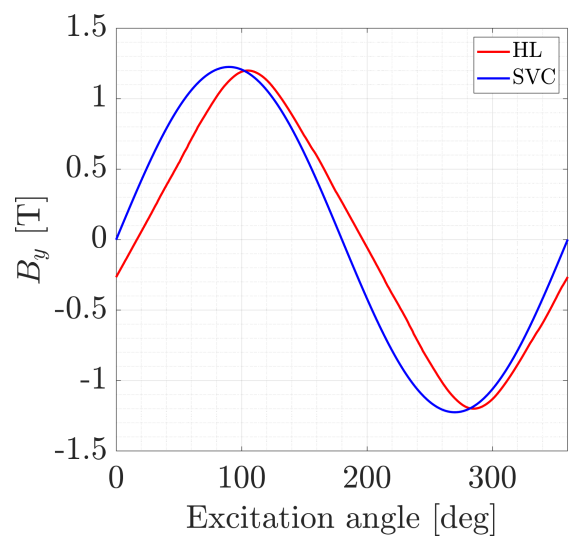

(b)

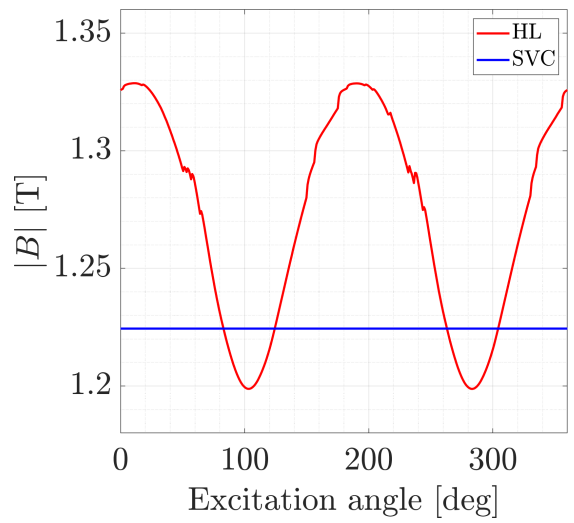

(c)

Fig. 3: Comparison of the magnetic flux densities by the models with SVC and HL: (a) $B_{x}$, (b) $B_{y}$, (c) $|B|$.

\section{RESULTS}

A single 2-D square-shaped MEC element with $1 \mathrm{~mm}$ edge and stack lengths is selected as the benchmark. All four branches of the MEC element are connected to the ground as in Fig 1. NO27 electrical steel is used as the material of the MEC element. SVC of $\mathrm{NO} 27$ is measured experimentally for different excitation directions. The average of the measured curves is used as the SVC in this study. To verify the developed loopbased MEC model and the fixed-point algorithm, the MEC model with SVC is simulated under different excitation levels. Fig. 2(a) shows that simulation results of the model agree with the SVC of the soft-magnetic material. After the verification of the MEC model, a rotating magnetic field with $200 \mathrm{~A} / \mathrm{m}$ magnitude and $50 \mathrm{~Hz}$ frequency $\left(\vec{H}_{i n}=200 \angle(2 \pi 50 t)[\mathrm{A} / \mathrm{m}]\right)$ is applied to both models with SVC and HL. The comparison of their convergence behaviors for a random time instant is presented in Fig. 2(b). Finite accuracy on calculating the weight function of the HL makes the convergence of
HL slower than SVC. Also, any error from the previous time step propagates to the current time step in HL, while time steps are independent of each other for the model with SVC. Although the convergence of the SVC is similar for each time step for the rotating magnetic field excitation with a constant magnitude, the angle of the applied magnetic field affects the convergence of HL as shown in Fig. 2(c). Bi-directional excitation such as 45 and 135 deg decreases the convergence rate of the non-linear solver for the model with HL. Fig. 3 shows the variation of $B_{x}, B_{y}$, and $|B|$ with respect to the excitation angle for the same rotating excitation with the previous case. It is observed that $B_{x}$ and $B_{y}$ calculated by SVC and HL have different peak values and phase angles. Moreover, the resultant $|B|$ of $\mathrm{HL}$ has a ripple in its flux density, while SVC gives a constant value.

In addition to the rotating magnetic field case, the alternating magnetic field is also applied to the MEC element. In Fig. 4, the flux density variations calculated by two methods are compared for alternating magnetic field 


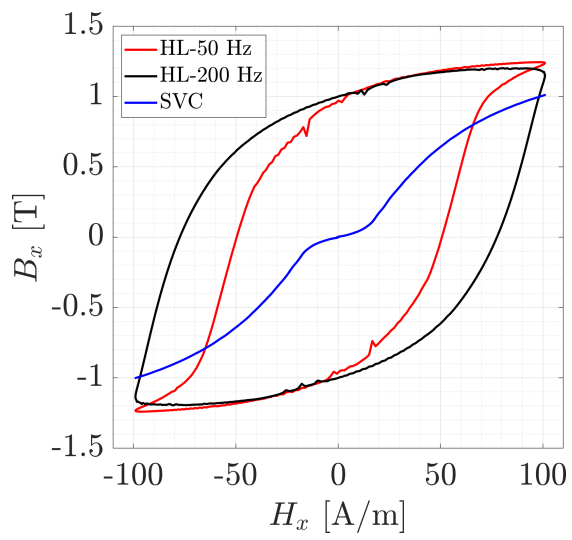

(a)

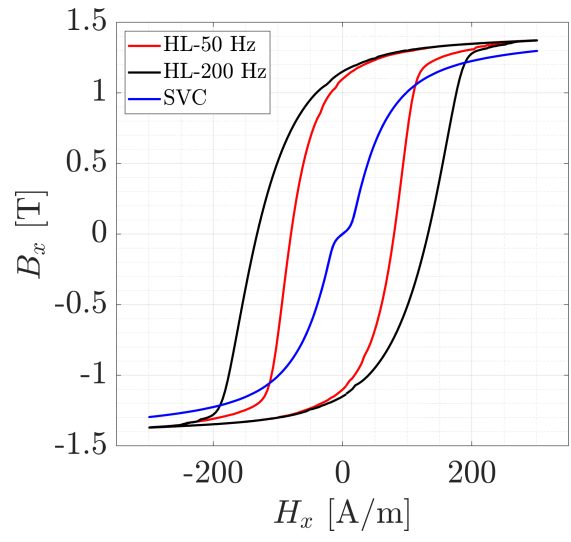

(b)

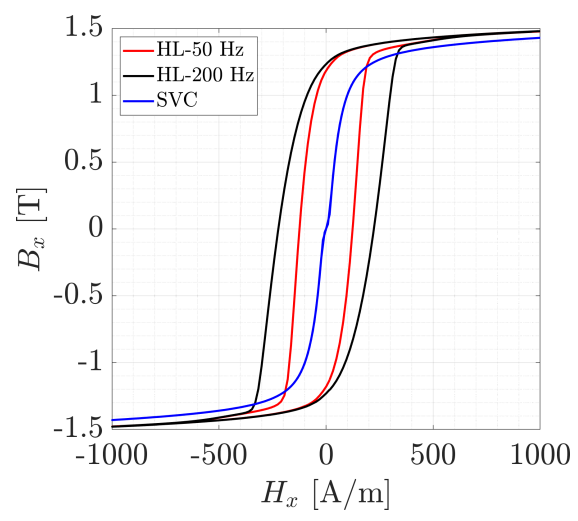

(c)

Fig. 4: Comparison of the magnetic flux density variations calculated by the models with SVC and HL for the alternating excitation with different magnitudes and frequencies (a) $\widehat{H}_{i n}=100 \mathrm{~A} / \mathrm{m}$, (b) $\widehat{H}_{i n}=300 \mathrm{~A} / \mathrm{m}$, (c) $\widehat{H}_{i n}=1000 \mathrm{~A} / \mathrm{m}$.

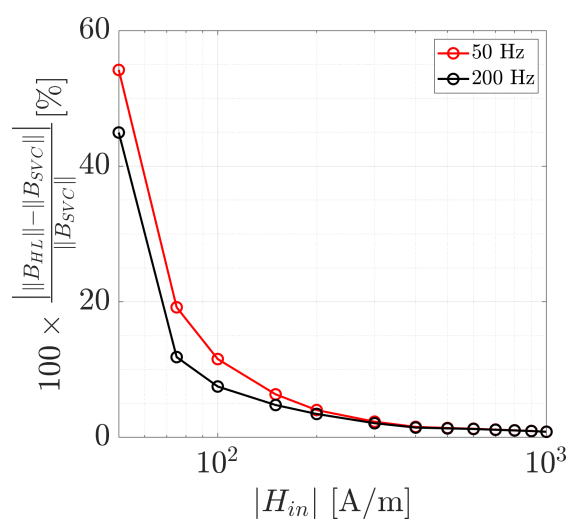

(a)

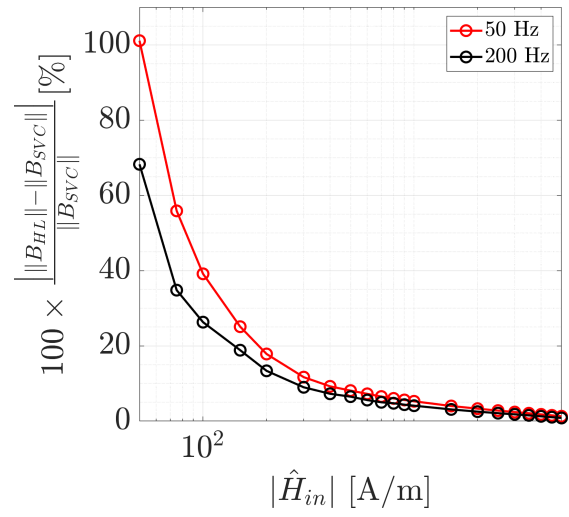

(b)

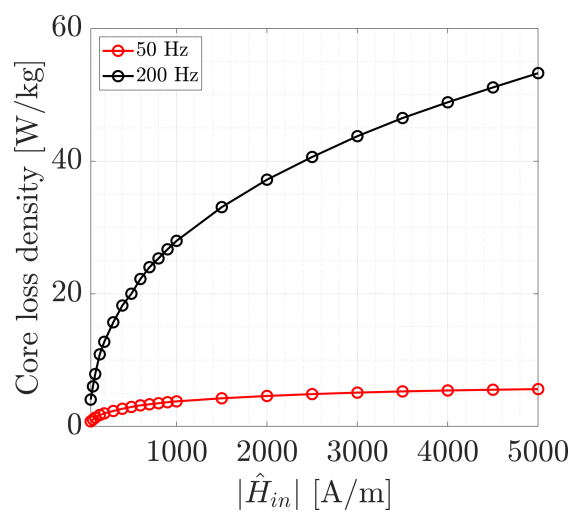

(c)

Fig. 5: Discrepancy between the RMS magnetic flux densities calculated by the models with SVC and HL for: (a) the rotating magnetic field excitation, (b) the alternating magnetic field excitation. (c) Variation of the core loss density for the alternating magnetic field excitation with different magnitudes and frequencies calculated by the model with HL.

excitations in $x$-direction with 100,300 , and $1000 \mathrm{~A} / \mathrm{m}$ peak values; and 50 and $100 \mathrm{~Hz}$ frequencies. It is indicated that applying a larger magnetic field magnitude decreases the difference between the results of SVC and HL. Moreover, increasing the alternating frequency of the excitation widens the operating hysteresis loop.

To indicate the difference between $|B|$ outputs of the models with SVC and HL, the root-mean-square (RMS) of $|B|$ in one excitation period is calculated for both rotating and alternating cases. Fig. 5(a) presents the percentage discrepancy between the methods for the rotating magnetic field with different magnitudes, while Fig. 5(b) shows the same comparison for the alternating magnetic field with a fixed angle and different peak values. It is observed that the modeling HL is more critical for the alternating magnetic field than the rotating one since the discrepancy is generally larger for the alternating excitation, which is usually present in a tooth body. Moreover, Fig. 5(a) and (b) show that applying an excitation with a smaller frequency decreases the accuracy of the flux density calculation with the SVC. Although using the SVC is a valid approximation for the accurate flux density calculation under deep magnetic saturation, SVC is not able to estimate the iron loss. The total iron loss density of the MEC element is calculated by the model with HL using

$$
P=\frac{1}{T \rho} \int_{0}^{T} H(t) \frac{d B(t)}{d t} d t
$$

where $T$ is the excitation period, and $\rho$ is the mass density of the material which is $7650 \mathrm{~kg} / \mathrm{m}^{3}$ for NO27. The total iron loss consists of the hysteresis, eddy current, and excess losses. Fig. 5(c) presents the variation 
of the iron loss with respect to the peak value of the alternating magnetic field for two different frequencies. It is observed that both large peak value and frequency increase the total iron loss density. Therefore, it is concluded that although using SVC approximation in the magnetic saturation region provides accurate flux density calculation which also means an accurate torque calculation for rotating electrical machines, it cannot estimate the iron loss which causes an inaccurate efficiency calculation.

\section{Conclusion}

A new method to include the anisotropic vector hysteresis in the MEC modeling is presented in this study. Although the proposed method increases the required number of fixed-point iterations for the non-linear solver, the results show that using the vector hysteresis is critical especially for alternating magnetic fields with smaller peak value than $300 \mathrm{~A} / \mathrm{m}$, where the knee point of the used SVC is located around $200 \mathrm{~A} / \mathrm{m}$. Otherwise, using the SVC of the material decreases the accuracy of the flux density calculation by more than $10 \%$. Moreover, the proposed method with the vector hysteresis property of the soft-magnetic material is able to estimate the core loss density which is getting more critical to include in the simulation for larger excitation magnitudes and frequencies. The core loss density is found to be 4 and $28 \mathrm{~W} / \mathrm{kg}$ for an alternating magnetic field excitation with a peak value of $1000 \mathrm{~A} / \mathrm{m}$ and frequencies of 50 and $200 \mathrm{~Hz}$, respectively.

\section{REFERENCES}

[1] M. Bali, H. D. Gersem, and A. Muetze "Finite-element modeling of magnetic material degradation due to punching," IEEE Trans. Magn., vol. 50, no. 2, pp. 1-4, Feb. 2014.

[2] P. J. Leonard, P. Marketos, A. J. Moses, and M. Lu "Iron losses under PWM excitation using a dynamic hysteresis model and finite elements," IEEE Trans. Magn., vol. 42, no. 4, pp. 907910, Apr. 2006.

[3] H. Gholizad, B. Funieru, and A. Binder "Direct modeling of motional eddy currents in highly saturated solid conductors by the magnetic equivalent circuit method," IEEE Trans. Magn., vol. 45, no. 3, pp. 1-4, Mar. 2009.

[4] A. D. Brown, J. N. Ross, and K. G. Nichols "Time-domain simulation of mixed nonlinear magnetic and electronic systems," IEEE Trans. Magn., vol. 37, no. 1, pp. 522-532, Jan. 2001.

[5] S. Piersanti, E. Pellegrino, G. Tresca, F. Paulis, and A. Orlandi, "Equivalent circuit modeling in time domain of the hysteresis of magnetic materials," IEEE Trans. Electromagn. Compat., vol. 57, no. 5, pp. 1013-1020, Oct. 2015.

[6] S. F. Rabbi, and M. A. Rahman "Equivalent circuit modeling of a hysteresis interior permanent magnet motor for electric submersible pumps," IEEE Trans. Magn., vol. 52, no. 7, pp. 1-4, Jul. 2016.

[7] Y. Hane, and K. Nakamura "Dynamic hysteresis modeling for magnetic circuit analysis by incorporating play model and Cauer's equivalent circuit theory," IEEE Trans. Magn., vol. 56, no. 8, pp. 1-6, Aug. 2020.
[8] N. Li, J. Zhu, M. Lin, G. Yang, Y. Kong, and L. Hao "Analysis of axial field flux-switching memory machine based on 3-D magnetic equivalent circuit network considering magnetic hysteresis," IEEE Trans. Magn., vol. 55, no. 6, pp. 1-4, Jun. 2019.

[9] D. Ceylan, L. A. J. Friedrich, K. O. Boynov, and E. A. Lomonova "A new loop-based hybrid analytical modeling formulation and the selection of its nonlinear solver," IEEE Trans. Magn., early access, pp. 1-4, Feb. 2021.

[10] R. Zeinali, D. Krop, and E. A. Lomonova "Anisotropic congruency-based vector hysteresis model applied to nonoriented laminated steels," IEEE Trans. Magn., early access, pp. 1-4, Feb. 2021.

[11] D. Ceylan, L. A. J. Friedrich, K. O. Boynov, and E. A. Lomonova "Convergence analysis of the fixed-point method with the hybrid analytical modeling for 2-D nonlinear magnetostatic problems," IEEE Trans. Magn., vol. 57, no. 2, pp. 1-5, Sep. 2020.

[12] H. W. Derbas, J. M. Williams, A. C. Koenig, and S. D. Pekarek "A comparison of nodal- and mesh-based magnetic equivalent circuit models," IEEE Trans. Energy Convers., vol. 24, no. 2, pp. 388-396, Jun. 2009. 